\title{
A Symmetric Continuous Poker Model
}

\author{
A. J. Goldman and J. J. Stone ${ }^{1}$
}

(August 18, 1959)

\begin{abstract}
Beginning with von Neumann, mathematicians concerned with the rational analysis of conflict situations have realized that investigation of accurate or simplified versions of common card games leads to techniques and insights applicable to situations of military or economic interest. In the present paper, a symmetric poker model one stage more complicated than the original von Neumann game is solved. There are two bet levels, $a, b$, and an ante of 1 unit $(a>b>1)$; no raises are permitted. The game has a unique optimal strategy, which forbids bluffing on a low hand. The limiting case $b=1$ is shown to yield the von Neumann model.
\end{abstract}

\section{Introduction}

The technical literature of the mathematical theory of games of strategy can be roughly subdivided into (i) general papers, which deal with concepts, existence theorems and computational methods relevant for broad classes of games, and (ii) specific papers, in which the solutions of particular games are determined. Although many of the studies in the second category have dealt with problems suggested by economic or military conflicts, e.g. $[1,2]^{2}$, a substantial fraction have dealt with accurate or simplified versions of common card games, the best known example being von Neumann's analysis of a simple poker model ([3], sec. 19.4 to 19.10). Bridge and baccarat have been treated $[4,5]$, but poker has remained the favorite topic for these studies [3, 6 to 10$]$, perhaps because the poker models investigated have presented just the right degree of challenge ("not easy, but not impossibly hard") to the ingenuity of the analyst. Of course the techniques and insights developed in these investigations are then applicable to situations of greater practical importance.

The present paper deals with a symmetric poker game one stage more complicated than von Neumann's original model. The game is continuous in the sense that the difficulties [6] due to the enormous but finite number of possible hands have been avoided by assuming (as von Neumann did) an infinite continuum of possible hands. The (more difficult) asymmetric version has also been solved by the authors; the results will appear elsewhere [11].

\section{Description of the Game}

The rules of the game are as follows. The two players $R$ and $S$ first ante 1 unit. They then receive independent random numbers (hands) from the interval $[0,1]$. Each player knows his own hand, but not that of his opponent. The players act simultaneously, each either dropping, betting an additional

1 Present address, Stanford University.

2 Italicized figures in brackets indicate the literature references at the end of this paper.

$b-1$ units, or betting an additional $a-1$ units. If both drop, then play ends and no payment is made. If both bet $b-1$ units or both bet $a-1$ units, then play ends and the player with the higher hand ${ }^{3}$ wins the ante and bet of his opponent. If one player bets $a-1$ units and the other bets $b-1$, then the latter can either fold (in which case he loses his ante and previous bet to his opponent) or see ${ }^{4}$ by betting an additional $a-b$ units (in which case the player with the higher hand wins the ante and previous bet or bets of his opponent); in either case play ends. Naturally we assume $a>b>1$.

Each player has four courses of action for any given hand. These are (1) to drop, (2) to bet $b-1$ units with the intention of folding if the opponent bets $a-1$ units, (3) to bet $b-1$ units with the intention of seeing if the opponent bets $a-1$ units, and (4) to bet $a-1$ units. Figures 1 and 2 show the algebraic payoff from player $S$ to player $R$ for any choices of courses of action by the players.

A strategy (for either player) is a quadruple $r(z)=\left\{r_{i}(z)\right\} \quad(i=1,2,3,4)$ of functions defined for $0 \leq z \leq 1$. Since $r_{i}(z)$ represents the probability ${ }^{5}$ that the player will choose the $i$ th course of action when his hand is $z$, the restrictions

$$
r_{i}(z) \geq 0, \quad r_{1}(z)+r_{2}(z)+r_{3}(z)+r_{4}(z)=1
$$

are imposed. The "smoothness" conditions on these functions are (i) that they be integrable, (ii) that if $r_{i}\left(z^{\prime}\right)>0$ then, for any interval $J$ of which $z^{\prime}$ is an interior ${ }^{6}$ point,

$$
\int_{J} r_{i}(t) d t>0
$$

and finally ${ }^{7}$ (iii) that

$$
\int_{0}^{z}\left[b r_{2}(t)-a r_{4}(t)\right] d t=0 \quad \text { on } \quad[(b-1) / b,(a-1) / a]
$$

3 The possibility of equal hands has zero probability and so can be disregarded. 4 This possibility distinguishes our model from the case $m=3$ of the version

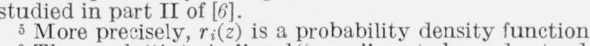

6 The words "interior" and "open" are to be understood relative to the interval $[0,1]$.

This very weak but ad hoc condition is precisely what is needed to prove (b) and (c) of lemma 8 . 
implies

$$
b r_{2}(z)-a_{4}(z)=0 \quad \text { on } \quad[(b-1) / b,(a-1) / a]
$$

It is convenient to define, for any $r(z)$,

$$
R_{i}=\int_{0}^{1} r_{i}(t) d t
$$

For any $r(z), G_{i}(z)$ will denote the expected payoff to player $R$, using $r(z)$, against a fixed hand $z$ and a fixed course of action $i$ of his opponent. Thus the expected payoff function of the game is given by

$$
K\left(r, r^{*}\right)=\int_{0}^{1} \sum r_{i}^{*}(z) G_{i}(z) d z
$$

\begin{tabular}{|c|c|c|c|c|}
\hline N & 1 & 2 & 3 & \\
\hline 1 & 0 & -1 & -1 & -1 \\
\hline 2 & 1 & $b$ & $b$ & $-b$ \\
\hline 3 & 1 & $b$ & $b$ & $a$ \\
\hline 4 & 1 & $b$ & $a$ & $a$ \\
\hline
\end{tabular}

The explicit formulas ${ }^{8}$ for the $G_{i}(z)$ are readily found from figures 1 and 2 :
FigURE 1

$R$ has the higher hand.

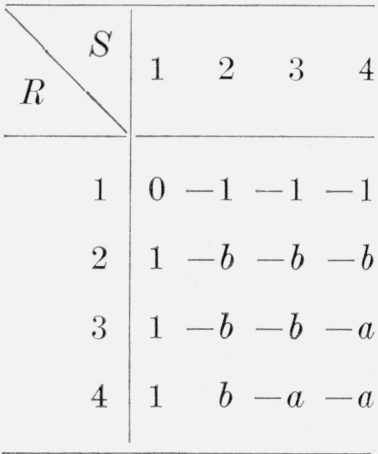

Figure 2

$S$ has the higher hand.

$$
\begin{aligned}
G_{1}(z)=\int_{0}^{z}\left[r_{2}(t)+r_{3}(t)\right. & \left.+r_{4}(t)\right] d t \\
& +\int_{z}^{1}\left[r_{2}(t)+r_{3}(t)+r_{4}(t)\right] d t,
\end{aligned}
$$

$$
\begin{aligned}
G_{2}(z)= & \int_{0}^{z}\left[-r_{1}(t)-b r_{2}(t)-b r_{3}(t)+b r_{4}(t)\right] d t \\
& +\int_{z}^{1}\left[-r_{1}(t)+b r_{2}(t)+b r_{3}(t)+b r_{4}(t)\right] d t
\end{aligned}
$$

$$
\begin{aligned}
G_{3}(z)= & \int_{0}^{z}\left[-r_{1}(t)-b r_{2}(t)-b r_{3}(t)-a r_{4}(t)\right] d t \\
& +\int_{z}^{1}\left[-r_{1}(t)+b r_{2}(t)+b r_{3}(t)+a r_{4}(t)\right] d t
\end{aligned}
$$

$$
\begin{aligned}
G_{4}(z)= & \int_{0}^{z}\left[-r_{1}(t)-b r_{2}(t)-a r_{3}(t)-a r_{4}(t)\right] d t \\
& +\int_{z}^{1}\left[-r_{1}(t)-b r_{2}(t)+a r_{3}(t)+a r_{4}(t)\right] d t .
\end{aligned}
$$

\footnotetext{
8 Note that the $\mathrm{G}_{i}(z)$ 's are shown by these formulas to be continuous functions.
}

\section{Plan of Attack}

It would not be very instructive simply to state the solution of the game and demonstrate its correctness. Instead, a slightly polished version of the process actually used to solve the game will be presented. This is done in sections 4 and 5 , in which it is proved (subject to the plausible assumption. stated in sec. 4) that the only possible optimal strategy is the one obeying the conditions (a) to (d) of lemma 8, which deal with the behavior of the strategy on three intervals $[0, g),\left(g, g^{\prime}\right)$, and $\left(g^{\prime}, 1\right]$ which might be thought of as the sets of low hands, intermediate hands, and high hands. It is then relatively easy to verify that this particular strategy is indeed the unique optimal strategy; this is done in section 6 , primarily by inspection, of the expected payoff function (2.1). In section 7 the limiting case $b=1$ is dealt with, and this game is found to have a unique optimal strategy obtained ${ }^{9}$ by "passing to the limit" in the optimal strategy for $b>1$. The von Neumann poker game of sections 19.4 to 19.10 of [3] is shown to arise as a "reduced game" from this limiting case.

In section 4 it is shown that $r_{3}(z)=0$ on $[0,1]$ for every optimal $r(z)$. The points $g, g^{\prime}$ are located, and the unique optimal $r(z)$ is isolated in section 5 . The arguments of these sections are essentially all applications of the following domination criterion:

The strategy $r(z)$ is optimal only if the following is true: For each z, $i$ for which $G_{i}(z)$ (as a function of $i$ ) does not assume its minimum, $r_{i}(z)=0$.

The correctness of this criterion follows from the smoothness condition (ii) and the fact that $r(z)$ is optimal only if

$$
K(r, r)=\min _{r^{*}} K\left(r, r^{*}\right) .
$$

\section{Elimination of One Course of Action}

The elimination is effected as follows.

Lemma 1. For every optimal $r(z), \quad r_{3}(z)=0$ on $[0,1]$.

Otherwise, for some optimal $r(z), R_{3}>0$ and $r_{3}(z)>0$ for some $z>0$. If $z^{\prime}$ is the least upper bound of the set of all such z, then by (2.4) and (2.5)

$$
G_{4}\left(z^{\prime}\right)-G_{3}\left(z^{\prime}\right)=-(a-b) R_{3}-2 b \int_{z^{\prime}}^{1} r_{2}(t) d t<0,
$$

so that by continuity and the domination criterion $r_{3}(z)=0$ in a neighborhood of $z^{\prime}$, contradicting the definition of $z^{\prime}$.

The following plausible assumption (which will be verified in sec. 6 ) is now made:

Assumption: For every optimal $r(z), \quad r_{\mathbf{4}}(1)>0$ (and thus $R_{4}>1$ ).

\footnotetext{
9 This is not quite the case for the asymmetric version see' 11].
} 


\section{Derivation of the Necessary Conditions}

Since application of the domination criterion requires examining the signs of the pairwise differences of the $G_{i}(z)^{\prime} s$, the formulas for these differences (based on (2.2) to (2.5) and the condition $r_{3}(z)=0$ on $[0,1])$ will first be given:

$$
\begin{array}{r}
G_{2}(z)-G_{1}(z)=(b-1)-b R_{1}-2 b \int_{0}^{z} r_{2}(t) d t \\
G_{3}(z)-G_{1}(z)=b R_{2}+a R_{4}-1-2 b \int_{0}^{z} r_{2}(t) d t \\
-2 a \int_{0}^{z} r_{4}(t) d t \\
G_{3}(z)-G_{2}(z)=(a-b) R_{4}-2 a \int_{0}^{z} r_{4}(t) d t \\
G_{4}(z)-G_{1}(z)=-1-b R_{2}+a R_{4}-2 a \int_{0}^{z} r_{4}(t) d t \\
G_{4}(z)-G_{2}(z)=-2 b R_{2}+(a-b) R_{4}-2 b \int_{0}^{z} r_{2}(t) d t \\
-2 a \int_{0}^{z} r_{4}(t) d t \\
G_{4}(z)-G_{3}(z)=-2 b R_{2}+2 b \int_{0}^{z} r_{2}(t) d t
\end{array}
$$

The first four of these continuous functions are clearly monotone nonincreasing. The following table ${ }^{10}$ defines the maximal open intervals in which these functions are positive $(+)$, zero $(0)$, and negative $(-)$. For each interval in the $(+)$ and $(-)$ columns, the result of applying the domination criterion is given. Inspection of (5.1) to (5.4) reveals conditions on the intervals in the $(0)$ column which are given below the interval. A question mark means that the interval may (so far as is known at the moment) be vacuous or degenerate to a single point; the condition written below such an interval is to apply only if the interval is nondegenerate.

The next three lemmas yield the values of $R_{1}, R_{2}$ and $R_{4}$ for optimal $r(z) \quad$ (lemma 1 shows that $R_{3}=0$ ).

Lemma 2. For every optimal $r(z)$ :

$$
\begin{aligned}
& G_{2}(0)-G_{1}(0)=(b-1)-b R_{1} \geq 0 . \\
& G_{4}(0)-G_{1}(0)=-1-b R_{2}+a R_{4} \leq 0 .
\end{aligned}
$$

If (a) were false, then the monotonicity of $G_{2}(z)-G_{1}(z)$ and the domination criterion would imply $R_{1}=0$, so that (a) would be true. If (b) were false, then since $G_{4}(1)-G_{1}(1)<0$ one would have

$$
G_{4}\left(z^{\prime}\right)-G_{1}\left(z^{\prime}\right)=0
$$

for some $z^{\prime}$ in $(0,1)$; by $(5.10) r_{4}(z)=0$ on $\left[0, z^{\prime}\right)$; so by (5.4)

$$
G_{4}(0)-G_{1}(0)=G_{4}\left(z^{\prime}\right)-G_{1}\left(z^{\prime}\right)=0
$$

and (b) would be true.

Lemma 3. For every optimal $r(z)$ :

$$
\begin{aligned}
& G_{2}(0)-G_{1}(0)=(b-1)-b R_{1}=0 . \\
& G_{4}(0)-G_{1}(0)=-1-b R_{2}+a R_{2}=0 .
\end{aligned}
$$

If $G_{2}(1)-G_{1}(1)>0$. then the monotonicity of $G_{2}(z)-G_{1}(z)$ and the domination criterion would imply $R_{2}=0$, so that on the one hand the initial assumption would read

$$
(b-1)-b R_{1}>0,
$$

\begin{tabular}{|c|c|c|c|}
\hline Difference & $(+)$ & $(0)$ & $(-)$ \\
\hline$G_{2}(z)-G_{1}(z)$ & {$\left[0, q_{-}^{21}\right) ?$} & $\left(g_{-}^{21}, g_{+}^{21}\right) ?$ & $\left(g_{+}^{21}, 1\right] ?$ \\
& $r_{2}(z)=0$ & $r_{2}(z)=0$ & $r_{1}(z)=0$ \\
\hline$G_{3}(z)-G_{1}(z)$ & {$\left[0, g_{-}^{31}\right) ?$} & $\left(g_{-}^{31}, g_{+}^{31}\right) ?$ & $\left(g_{+}^{31}, 1\right]$ \\
& $r_{3}(z)=0$ & $r_{1}(z)=1$ & $r_{1}(z)=0$ \\
\hline$G_{3}(z)-G_{2}(z)$ & {$\left[0, g_{-}^{32}\right)$} & $\left(g_{-}^{32}, g_{+}^{32}\right) ?$ & $\left(g_{+}^{32}, 1\right]$ \\
& $r_{3}(z)=0$ & $r_{4}(z)=0$ & $r_{2}(z)=0$ \\
\hline$G_{4}(z)-G_{1}(z)$ & {$\left[0, g_{-}^{41}\right) ?$} & $\left(g_{-}^{41}, g_{+}^{41}\right) ?$ & $\left(g_{+}^{41}, 1\right]$ \\
& $r_{4}(z)=0$ & $r_{4}(z)=0$ & $r_{1}(z)=0$ \\
\hline
\end{tabular}


and on the other hand $R_{1}=1-R_{4}$ (by lemma 1, using $R_{2}=0$ ). Combining these statements yields $R_{4}>1 / b$, which together with (5.4) and $R_{2}=0$ yields

$$
G_{4}(0)-G_{1}(0)=-1+a R_{4}>0,
$$

contradicting (b) of lemma 2. Thus $G_{2}(1)-G_{1}(1) \leq 0$, which together with (a) of lemma 2 implies that

$$
G_{2}\left(z^{\prime}\right)-G_{1}\left(z^{\prime}\right)=0
$$

for some $z^{\prime}$ in $[0,1]$. By $(5.7) r_{2}(z)=0$ on $\left[0, z^{\prime}\right)$; so by (5.1),

$$
G_{2}(0)-G_{1}(0)=G_{2}\left(z^{\prime}\right)-G_{1}\left(z^{\prime}\right)=0,
$$

and (a) is proved. If (b) were false, then by (b) of lemma 2 , the monotonicity of $G_{4}(z)-G_{1}(z)$, and the domination criterion, we would have $R_{1}=0$, contradicting (a).

Lemma 4. For every optimal $r(z)$ :

$$
\begin{array}{ll}
\text { (a) } & R_{1}=(b-1) / b . \\
\text { (b) } & R_{2}=(a-b) / b(a+b) . \\
\text { (c) } & R_{4}=2 /(a+b) .
\end{array}
$$

This follows from lemma 3 and the corollary $R_{1}+R_{2}+R_{4}=1$ of lemma 1 .

Next the points $g, g^{\prime}$ will be located, and the behavior of an optimal $r(z)$ on $[0, g),\left(g, g^{\prime}\right)$ and $\left(g^{\prime}, 1\right]$ determined.

Lemma 5. For every optimal $r(z)$ :

$$
\begin{array}{lll}
\text { (a) } & g_{+}^{21}=g_{+}^{41}=g=(b-1) / b . \\
\text { (b) } & r_{1}(z)=1 & \text { on }[0, g) . \\
\text { (c) } & r_{1}(z)=0 & \text { on }(g, 1] .
\end{array}
$$

By (5.5) and lemma 4,

$$
G_{4}(z)-G_{2}(z)=2 b \int_{0}^{z} r_{2}(t) d t-2 a \int_{0}^{z} r_{4}(t) d t
$$

If $g_{+}^{21}<g_{+}^{41}$, then by $(5.7)$ and $(5.10)$ one has $r_{2}(z)=1$ on $\left(g_{+}^{21}, g_{+}^{41}\right), r_{1}(z)=1$ on $\left[0, g_{+}^{21}\right)$, and $r_{4}(z)=0$ on $\left[0, g_{+}^{41}\right)$, so that the last equation yields

$$
G_{4}\left(g_{+}^{41}\right)-G_{2}\left(g_{+}^{41}\right)>0 ;
$$

continuity and the domination criterion show that $r_{4}(z)=0$ in a neighborhood of $g_{+}^{41}$, but (5.4) and (b) of lemma 3 show that this contradicts the definition of $g_{+}^{41}$. An entirely similar argument refutes the possibility $g_{+}^{41}<g_{+}^{21}$, and so $g_{+}^{21}=g_{+}^{41}$. By (5.7) and (5.10) we have $r_{1}(z)=1$ on $\left[0, g_{+}^{21}\right)$ and $r_{1}(z)=0$ on $\left(g_{+}^{21}, 1\right]$, so that $g_{+}^{21}=R_{1}$; an application of (a) of lemma 4 completes the proof.
Leman 6. For every optimal $r(z)$ :
(a) $g_{+}^{32}=a^{\prime}=(a-1) / a$.
(b) $r_{4}(z)=1$ on $\left(g^{\prime}, 1\right]$.

Note first that $r_{4}(z)=1$ on $\left(g_{+}^{32}, 1\right]$ since (i) $r_{2}(z)=0$ on $\left(g_{+}^{32}, 1\right]$ by $(5.9)$, (ii) $r_{1}(z)=0$ on $(g, 1]$ by lemma 5 , and (iii) $g<g_{+}^{32}$ because $G_{3}(g)-G_{2}(g)>0$ by (5.3) and the fact (see $(5.10)$ ) that $r_{4}(z)=0$ on $[0, g)$. It follows that

$$
\int_{0}^{{ }^{32}} r_{4}(t) d t+\left(1-g_{+}^{32}\right)=R_{4}=2 /(a+b) .
$$

The definition of $g_{+}^{32}$ together with (5.3) shows that

$$
\int_{0}^{g_{+}^{32}} r_{4}(t) d t=(a-b) R_{4} / 2 a=(a-b) / a(a+b),
$$

and combining the last equations yields the asserted value of $g_{+}^{32}$.

Lemma 7. For every optimal $r(z)$,

$$
G_{4}(z)-G_{2}(z)=0 \quad \text { on }\left[g, g^{\prime}\right] .
$$

Since $r_{2}(z)=0$ on $\left(g^{\prime}, 1\right],(5.11)$ and (5.12) imply

$$
G_{4}\left(g^{\prime}\right)-G_{2}\left(g^{\prime}\right)=2 b R_{2}-(a-b) R_{4}=0,
$$

where the last equality follows from lemma 3. Let $z^{*}$ be a point at which $G_{4}(z)-G_{2}(z)$ attains its maximum $M$ on $\left[g, g^{\prime}\right]$; the last equation shows that $M \geq 0$. If $M>0$, then by continuity and the domination criterion one has $r_{4}(z)=0$ (and thus $r_{2}(z)=1$, since $r_{1}(z)=0$ on $\left.(g, 1]\right)$ on some interval $\left(z^{*}, z^{\prime}\right)$; by $(5.5)$ $G_{4}(z)-G_{2}(z)$ is strictly increasing on $\left(z^{*}, z^{\prime}\right)$, which contradicts the definition of $z^{*}$ unless $z^{*}=g^{\prime}$, in which case $M=0$ by the last equation. Thus $M=0$; an entirely similar argument shows that the minimum value of $G_{4}(z)-G_{2}(z)$ on $\left[g, g^{\prime}\right]$ is $m=0$, and so the lemma is proved.

The completion and summary of the preceding material is as follows.

Lemma 8. Let $g=(b-1) / b$ and $g^{\prime}=(a-1) / a$. If the assumption at the end of section 4 is correct, then the only possible optimal strategy is the strategy defined by
(a) $\quad r_{1}(z)=1$ on $[0, g)$.$$
r_{2}(z)=a /(a+b) \text { on }\left(g, g^{\prime}\right) \text {. }
$$
(d)

$$
\begin{array}{ll}
r_{4}(z)=b /(a+b) & \text { on }\left(g, g^{\prime}\right) . \\
r_{4}(z)=1 & \text { on }\left(g^{\prime}, 1\right] .
\end{array}
$$

From lemma 1, lemma 5, and lemma 6 one has (a), (d), and also

$$
r_{2}(z)+r_{4}(z)=1 \quad \text { on }\left(g, g^{\prime}\right) .
$$


From lemma $7,(5.5)$, and lemma 4 one has

$$
\int_{0}^{z}\left[b r_{2}(t)-a r_{4}(t)\right] d t=0 \quad \text { on } \quad\left(g, g^{\prime}\right),
$$

which by smoothness condition (iii) (see section 2) implies

$$
b r_{2}(z)-a r_{4}(z)=0 \quad \text { on }\left(g, g^{\prime}\right) ;
$$

this and (5.13) imply (b) and (c).

\section{Proof That the Conditions Are Necessary and Sufficient for Optimality}

In this section the notation $r(z)$ is reserved for the particular strategy described in lemma 8 , while arbitrary strategies are denoted $r^{*}(z)$.

Lemia 9. $K\left(r, r_{0}^{*}\right)=\min K\left(r, r^{*}\right)$ if and only if $r_{0}^{*}(z)$ obeys

$$
\begin{aligned}
& \text { (a) } \quad r_{3}^{*}(z)=0 \quad \text { on }[0, g) \text {. } \\
& \text { (b) } \quad r_{1}^{*}(z)=r_{3}^{*}(z)=0 \quad \text { on }\left(g, g^{\prime}\right) \text {. } \\
& \text { (c) } \quad r_{1}^{*}(z)=r_{2}^{*}(z)=0 \quad \text { on }\left(g^{\prime}, 1\right] \text {. }
\end{aligned}
$$

The $K\left(r, r^{*}\right)$ of $(2.1)$ is first written as the sum of these integrals:

$$
\begin{aligned}
& \int_{0}^{g}\left[G_{1}(z)+r_{2}^{*}(z)\left(G_{2}(z)-G_{1}(z)\right)+r_{3}^{*}(z)\left(G_{3}(z)\right.\right. \\
& \left.\left.-G_{1}(z)\right)+r_{4}^{*}(z)\left(G_{4}(z)-G_{1}(z)\right)\right] d z . \\
& \int_{G}^{g^{\prime}}\left[G_{2}(z)+r_{1}^{*}(z)\left(G_{1}(z)-G_{2}(z)\right)+r_{3}^{*}(z)\left(G_{3}(z)\right.\right. \\
& \left.\left.-G_{2}(z)\right)+r_{4}^{*}(z)\left(G_{4}(z)-G_{2}(z)\right)\right] d z . \\
& \int_{q^{\prime}}^{1}\left[G_{4}(z)+r_{1}^{*}(z)\left(G_{1}(z)-G_{4}(z)\right)+r_{2}^{*}(z)\left(G_{2}(z)\right.\right. \\
& \left.\left.-G_{4}(z)\right)+r_{3}^{*}(z)\left(G_{3}(z)-G_{4}(z)\right)\right] d z .
\end{aligned}
$$

It is easily verified that $r(z)$ has the properties described in lemma 4 , and since $r_{3}(z)=0$ on $[0,1]$, $r(z)$ obeys $(5.1)-(5.6)$. Using these facts, one finds that on $[0, g)$ the coefficients of $r_{2}^{*}(z)$ and $r_{4}^{*}(z)$ in the first integral vanish, while that of $r_{3}^{*}(z)$ is positive. Hence $r_{0}^{*}(z)$ minimizes the first integral if and onl if it satisfies (a). Similarly, $r_{0}^{*}(z)$ minimizes the second integral if and only if it satisfies (b), and minimizes the third integral if and only if it satisfies (c).

\section{THEOREM. $r(z)$ is the unique optimal strategy.}

Since $r(z)$ obeys $(a)$ to $(c)$ of lemma 9 ,

$$
K(r, r)=\min _{r^{*}} K\left(r, r^{*}\right)
$$

which (since the game is symmetric) implies that $r(z)$ is optimal. Thus any optimal $r_{0}^{*}(z)$ must satisfy

$$
K\left(r, r_{0}^{*}\right)=\min _{r^{*}} K\left(r, r^{*}\right)
$$

and thus, by lemma 1 and (c) of lemma 9, must satisfy $r_{4}^{*}(1)=1$. Hence the assumption at the end of section 4 is correct, and so, by lemma $8, r(z)$ is the only optimal strategy.

\section{Discussion of a Limiting Case}

The limiting case $b=1$ will now be briefly discussed. (Recall that $b>1$ previously.) It is easily verified that the results of the previous sections remain valid (many of the proofs can be considerably simplified), so that the game with $b=1$ has a unique optimal strategy which is obtained from that for $b>1$ (i.e., from (a) to (d) of lemma 8) by setting $b=1$. Since $g=0$ for $b=1$, the unique optimal strategy is given by

$$
\begin{array}{llll}
\text { (a) } & r_{2}(z)=a /(a+1) & \text { on } & {[0,(a-1) / a) \text {. }} \\
\text { (b) } & r_{4}(z)=1 /(a+1) & \text { on } & {[0,(a-1) / a)} \\
\text { (c) } & r_{4}(z)=1 & \text { on } & ((a-1) / a, 1] .
\end{array}
$$

Upon changing

$$
\begin{aligned}
& \text { player } R \text {, player } S, r_{2}, r_{3}, r_{4}, a \\
& \text { to player } 1 \text {, player } 2, \rho_{3}, \rho_{2}, \rho_{1}, a / b
\end{aligned}
$$

respectively, one finds that this strategy is precisely the unique optimal strategy of the continuous von Neumann poker game discussed in sections 19.4 to 19.10 of [3]. To explain this, note that (2.2) and $(2.3)$, with $b=1$, vield

$$
G_{2}(z)-G_{1}(z)=-R_{1}-2 \int_{0}^{z}\left[r_{2}(t)+r_{3}(t)\right] d t \leq 0
$$

for all strategies $r(z)$, which on the one hand shows that the first course of action is dominated, and on the other hand yields a quick proof ${ }^{11}$ that $r_{1}(z)=0$ on $[0,1]$ for all optimal $r(z)$. Thus, without loss of optimal strategies, the game can be "reduced" by "striking out" the first course of action for both players. This reduced game is easily seen to be identical with the von Neumann game, except for some changes of language and a multiplicative factor $b$ in the expected payoff function. Thus the von Neumann game appears as a reduced form of the limiting case $b=1$ of the game solved in this paper.

11 If $r_{1}\left(z^{\prime}\right)>0$ for some $z^{\prime}$, then $R_{1}>0$; the formula and the domination eriterion yield $r_{1}(z)=0$ for all $z$, a contradiction. 


\section{References}

[1] L. E. Dubins, A discrete evasion game, Ann. Math. Study 39, 231-255 (1957).

[2] M. Shubik, Edgeworth market games, Ann. Math. Study 40, 267-278 (1959).

[3] J. von Neumann and O. Morgenstern, Theory of games and economic behavior (3d ed., Princeton Univ. Press, Princeton, N.J., 1953).

[4] G. L. Thompson, Bridge and signalling, Ann. Math. Study 28, 279-289 (1953)

[5] J. G. Kemeny, L. Snell, and G. L. Thompson, Gametheoretic solution of baccarat, Am. Math. Monthly 64, 465-469 (1957).
[6] D. B. Gillies, J. P. Mayberry, and J. von Neumann, Two variants of poker, Ann. Math. Study 28, 13-50 (1953).

[7] H. Kuhn, A simplified two-person poker, Ann. Math. Study 24, 97-103 (1950).

[8] J. Nash and L. S. Shapley, A simple three-person poker game, Ann. Math. Study 24, 105-116 (1950).

[9] R. Bellman, On games involving bluffing, Rend. Circ. Mat. Palermo [\%] 1, 139-156 (1952).

[10] S. Karlin and R. Restrepo, Multistage poker models, Ann. Math. Study 39, 337-363 (1957).

[11] A. J. Goldman and J. J. Stone, A continuous poker game, to appear in Duke Math. J. (1960).

Washington, D.C.

(Paper 64B1-18) 